Part of Journal of Research of the National Bureau of Standards, Volume 28, April 1942

\title{
ELECTRICAL CONDUCTION IN THE GLASS INSULATION OF RESISTANCE THERMOMETERS
}

\author{
By Harold J. Hoge
}

\begin{abstract}
At temperatures above $350^{\circ} \mathrm{C}$, electrical conduction may become troublesome in the glass through which the leads of certain types of resistance thermometers are sealed. It was found that conduction in these seals is rapidly reduced by polarization of the glass, and that the error in temperature measurement resulting from conduction in the glass may be considerably reduced by adopting a measuring schedule which takes full advantage of this polarization.

One type of glass was investigated under experimental conditions which prevented polarization. At $445^{\circ} \mathrm{C}$ this glass had a volume resistivity of approximately $21,000 \mathrm{ohm}-\mathrm{cm}$. The temperature dependence of the resistance could be represented very roughly by $1 / r=A e^{-a / k T}$, with $\epsilon$ having a value of the order of 0.8 electron volt.
\end{abstract}

\section{CONTENTS}

Page

I. Introduction

II. Experimental results

III. Supplementary observations

IV. References_._.

\section{INTRODUCTION}

The form of platinum resistance thermometer which consists of a Meyers-type coiled filament enclosed in a small platinum case was first used by Southard and Milner [1]. ${ }^{1}$ The coil is sealed inside the platinum case with a soft glass seal, as shown in figure 1 .

Although this type of thermometer is not ordinarily used above $100^{\circ} \mathrm{C}$, it is calibrated in the usual way [2] at the sulfur, steam, oxygen, and ice points. At the sulfur point $\left(444.60^{\circ} \mathrm{C}\right)$ the soft glass is noticeably conducting, the conduction taking place between the two pairs of leads which pass through the glass and connect the thermometer with the bridge or potentiometer. For most of our thermometers the electromotive force between the two pairs of leads at the sulfur point is of the order of $0.075 \mathrm{v}$, the measuring current being $1 \mathrm{ma}$ and the resistance at this temperature approximately $75 \mathrm{ohms}$. The conduction decreases as the temperature is lowered, and is not appreciable below $300^{\circ} \mathrm{C}$. It might pass unnoticed except for the very rapid polarization of the glass, which causes the measured resistance to increase as soon as the electromotive force is applied to the specimen. After the current has flowed for a few minutes, the rate of increase of

\footnotetext{
1 Figures in brackets indicate the literature references at the end of this paper.
} 
resistance becomes negligible, indicating that the current in the glass is approaching a limiting value.

The present investigation was undertaken to see whether or not the remaining current in the glass was large enough to cause appreciable error in temperature measurements. Three seals, as nearly as possible like those in figure 1 , were made, but with the thermometer coil omitted to make it possible to measure the resistance of the glass directly. A number of preliminary experiments on more roughly constructed seals had shown that, in general, the resistance of any specimen depended on its past thermal and electrical history. Accordingly, the

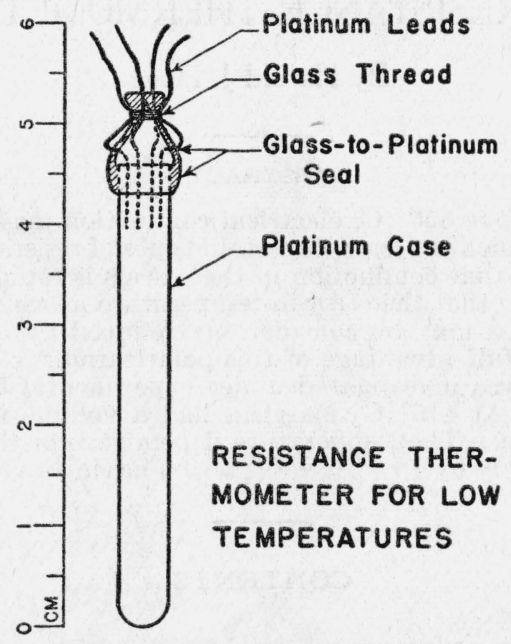

Figure 1.-Scale drawing of a thermometer.

The platinum leads are $0.2 \mathrm{~mm}$ in diameter. The platinum case has an inside diameter of $5 \mathrm{~mm}$ and weighs approximately $0.51 \mathrm{~g} / \mathrm{cm}$ of length. The bottom is spun to a hemispherical form and the center welded. After the thermometer is filled with helium and sealed off, the glass tip is flattened to provide a shoulder so that the leads may be tied down with glass thread.

three seals were subjected to identical test schedules. No electromotive forces were applied to any of them prior to the measurements reported.

\section{EXPERIMENTAL RESULTS}

The first group of experiments was made with an apparatus which did not duplicate exactly the conditions of use in resistance thermometry, but provided somewhat greater sensitivity and flexibility. These experiments showed the general nature of the conduction phenomena and the relative merits of the three types of glass. They are most readily explained by reference to figure 2 . A seal was placed in a small furnace and connected to the measuring equipment. Prior to the time $t=0$ the furnace and seal were held at a temperature of approximately $147^{\circ} \mathrm{C}$, but at this time the furnace current was suddenly raised to a higher value. The resulting rise in temperature of the seal was indicated by a Chromel-Alumel thermocouple, whose electromotive force is given by the lowest curve of figure 2 .

A $2,100-0 h m$ resistance was connected in series with the seal. Measurement of the potential drop across part of this allowed the current through the seal to be computed, while the remainder of the resist- 
ance served as a partial protection to the galvanometer in case of a short circuit in the seal. At regular intervals an electromotive force of $0.075 \mathrm{v}$ was applied to the seal and the galvanometer deflection noted after $4 \frac{1}{2}$ seconds, which was the time required to reach maximum deflection. Connections to the seal were reversed before each succeeding application of the electromotive force, to reduce the effects of polarization. The current through the seal in arbitrary units (millimeters galvanometer deflection) is plotted in the upper curves

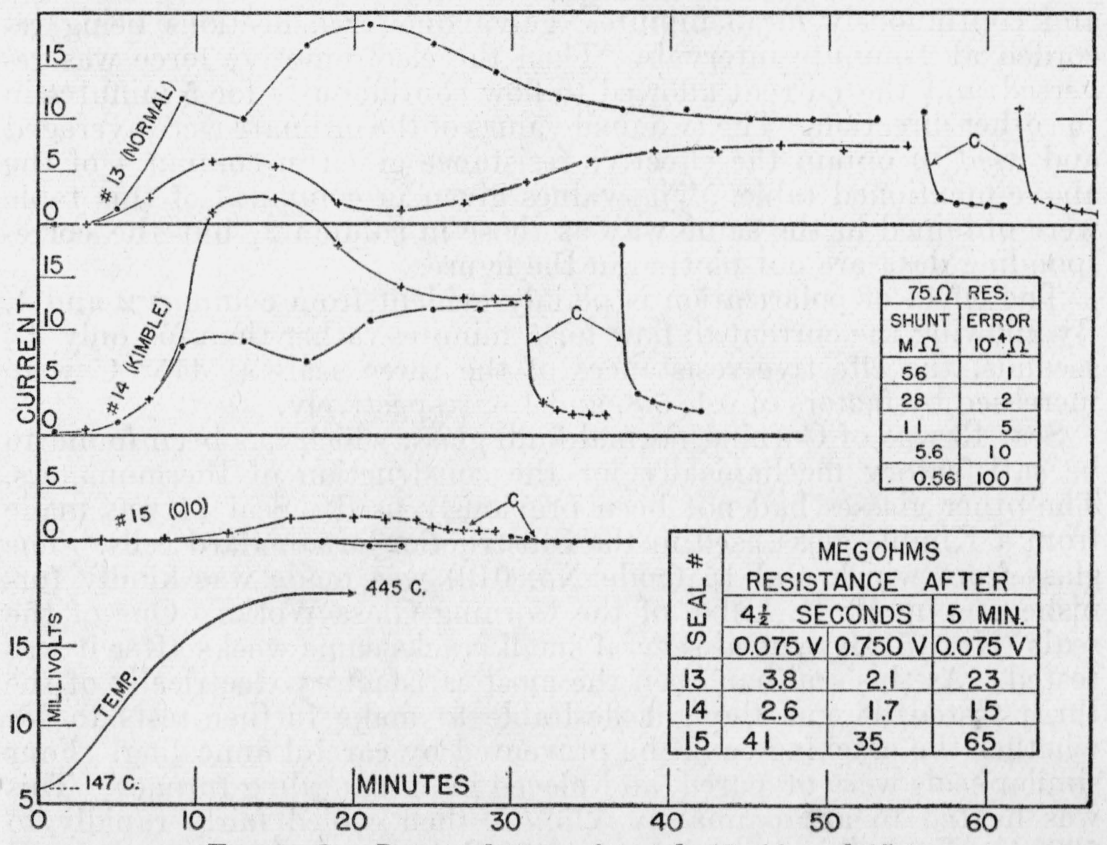

Figure 2.-Data and curves for seals 13, 14, and 15.

The current flowing through each seal was measured at intervals while the temperature was being raised. The six upper curves show the magnitude of this current in terms of galvanometer defiections, while the corresponding temperature may be obtained from the lowest curve, which shows the electromotive force developed by a Chromel-Alumel thermocouple placed in the furnace beside the test specimen.

The table of values at the lower right-hand corner gives the effective resistance of each seal at $445^{\circ} \mathrm{C}$ under various conditions of measurement. The upper table shows the magnitude of the error which will result if the thermometer has a resistance of $75 \mathrm{ohms}$, for various values of the effective resistance of the shunt (glass seal).

of figure 2 , where dots and crosses refer respectively to the two directions of application of electromotive force to the seal. The applied electromotive force was measured by means of a potentiometer. As electromotive forces as high as $9 \mathrm{v}$ were sometimes employed, the range of the potentiometer was extended by means of a volt box.

The existence of electromotive forces in the seals themselves is indicated by the fact that the currents in the two directions were not equal. The differences tended to disappear as the measurements continued. When an approximately steady state had been reached, the ordinates of the two curves for each seal were averaged and the current passing through the seal was computed. Dividing the applied electromotive force by this current yields the values in column 2 of the lower table of figure 2. All the values in this table were obtained at a seal temperature of $445^{\circ} \mathrm{C}$. The current passing through a seal is a function both 
of the ordinary resistance of the seal, and of the electromotive forces in the seal which result from the previous passage of current, each of which changes with the conditions of measurement. Hence the values in the lower table of figure 2 are not to be considered as resistances in the ordinary sense but rather as "effective resistances," which are nothing more than the quotient of the applied electromotive force by the current observed under the given conditions of measurement.

Data for the parts of the curves marked $C$ were taken in a different way. Here the electromotive force was applied in the same direction and continuously for 5 minutes, galvanometer deflections being recorded at 1-minute intervals. Then the electromotive force was reversed and the current allowed to flow continuously for 5 minutes in the other direction. The two final values of the ordinate were averaged and used to obtain the effective resistance given in column 4 of the above-mentioned table. The values given in column 3 of this table were obtained in the same way as those in column 2 , but the corresponding data are not plotted in the figure.

The effect of polarization is plainly evident from columns 2 and 4 . By allowing the current to flow for 5 minutes rather than for only $4 \frac{1}{2}$ seconds, the effective resistances of the three seals at $445^{\circ} \mathrm{C}$ were increased by factors of $6.1,5.8$, and 1.6 , respectively.

Seal 13 was of Corning normal bulb glass, which has been found to be satisfactory mechanically for the construction of thermometers. The other glasses had not been previously used. Seal 14 was made from a Kimble glass used in the construction of standard cells. The glass from which seal 15 (code No. 010) was made was kindly furnished us by H. R. Lillie, of the Corning Glass Works. One of the seals (No. 15) developed several small cracks some weeks after it was tested. As this seal had been the most satisfactory electrically of the three tested, it was thought desirable to make further tests to see whether the cracking could be prevented by careful annealing. Four similar seals were prepared, and placed in an annealing furnace. This was heated to approximately $525^{\circ} \mathrm{C}$, then cooled fairly rapidly to $500^{\circ} \mathrm{C}$. From this point down to $340^{\circ} \mathrm{C}$ the temperature was reduced slowly, by lowering the setting of the thermoregulator in $0.2-\mathrm{mv}$ (5degree) steps, at intervals of not less than 10 minutes.

These seals remained at room temperature for $2 \frac{1}{2}$ months without developing any cracks. They were then subjected to rapid heating and cooling, but were not, however, carried above the sulfur point $\left(445^{\circ} \mathrm{C}\right)$. No cracks have developed as a result of this treatment, and we may conclude that seals made of this glass will, if carefully annealed, stand up under conditions as severe as those experienced in a sulfur-point calibration. If during the annealing of the platinum coil the thermometer is heated to a temperature much above $445^{\circ} \mathrm{C}$, slow cooling may be necessary to avoid danger of breaking the seal.

From figure 2 it can readily be concluded that $4 \frac{1}{2}$ seconds is too short a time to allow the current to flow, if it is desired to take full advantage of the increase in effective seal resistance caused by polarization. Just how long it will be profitable to wait cannot be accurately predicted from the curves $C$, however, because of the dependence of the behavior of a seal on its past history. The rate at which the current through a seal diminishes with time is influenced by its initial condition of polarization, and this will depend in practice on the measuring schedule adopted. 
To approximate the conditions of actual resistance thermometry, a circuit was employed in which the seal placed in a furnace at $445^{\circ} \mathrm{C}$ could be connected as a shunt across a 100-ohm standard resistor, which formed one arm of a Wheatstone bridge. The suitability of various mesauring schedules was investigated by determining the reduction in apparent resistance of the $100-\mathrm{ohm}$ standard which resulted from the presence of the shunt.

In one set of measurements the battery key was closed for a certain length of time and then opened for the same length of time, and the seal error investigated as a function of this interval. The shunting effect of the seal decreased with increasing interval rapidly at first, but beyond a period of $1 / 2$ minute on and $1 / 2$ minute off the decrease in error was small. Unless the galvanometer zero is unusually stable, it should not ordinarily be worth while to increase the on and off intervals to more than 45 seconds each.

In another series of measurements the total period was held constant at $1 \frac{1}{2}$ minutes, while the relative lengths of the on and off periods were varied. The most desirable operating conditions were found to be in the neighborhood of 50 percent on and 50 percent off, but the minimum is rather broad.

The measurements described in the preceding paragraphs of this section were made without reversal of the current in the bridge. Many investigators balance their bridges not by opening and closing the battery circuit but by reversing the direction of the current, since the reversal gives double the deflection produced by simply reducing the current to zero. The method of reversal can be used, and the errors due to the glass seals are substantially the same as with the on-off method, when the time between reversals is made equal to the time on or off. If the temperature of a resistance thermometer is changing, as it will in a sulfur bath while the barometer is rising or falling, the on-off method of balancing will usually be found more convenient.

In a previously published abstract [3] it was recommended that the electromotive force be always applied to the seal in the same direction. This recommendation was based on the observed fact that the current falls less rapidly to its limiting value in a seal which has been previously polarized by passage of current in the opposite direction than when the current has previously flowed in the same direction. In spite of this fact the current-reversal method of balancing gave seal errors no larger than the on-off method. The probable explanation is the following: The galvanometer of a bridge which is in balance will be deflected in a certain direction when one arm is shunted by a glass seal, because of the passage of current through the seal. If the applied electromotive force is removed, by opening the battery key, the seal will discharge itself, and the galvanometer zero will be displaced in the opposite direction by the current from the seal. Hence the total effect of the seal on the balance of the bridge is the sum of two separate effects, when balance is obtained by the on-off method. When balance is obtained by current reversal, however, the entire effect of the seal is simply that of the current passing through it at the time readings are taken. With on and off intervals equal to the interval between reversals, an "on" current following an off period will indeed fall to a lower value than one which follows a current reversal; but because of the effect of the seal in displacing the galva- 
nometer zero during the off period, this does not necessarily mean that the on-off method is preferable to the method of reversals.

Seal 14, when measured with on and off intervals of 30 seconds each, caused an error equal to that of a 20 -megohm shunt. When measured with current reversal at 30 -second intervals, the same seal caused approximately the same error. A sufficient number of readings was taken in each case to insure the presence of a steady state.

The effective resistance found for the seal with current reversals at 30 -second intervals is larger than the effective resistance shown in figure 2 for the same seal with one cycle of current reversals at 5 -minute intervals (curves $C$ ). The difference is doubtless due to differences in the condition of the seal in the two cases, although because of the greater polarization which takes place in the longer interval, it should not be assumed that current reversal at 30-second intervals would yield curves identical with the initial portions of the curves for 5 -minute intervals.

When resistance measurements are made with a Mueller bridge, lead resistance is eliminated by interchanging lead connections. This normally reverses the electromotive force applied to the thermometer and will interrupt an otherwise uniform measuring schedule. This may be of no consequence if plenty of time can be taken for the measurement, but if it is necessary to go from " $N$ " to " $\mathrm{R}$ " rapidly, it is desirable to place a reversing switch in the battery circuit and to operate this switch whenever the commutator is changed from " $N$ " to " $\mathrm{R}$ " or vice versa. The direction of the flow of current in the seal will then be independent of the position of the commutator. If a four-pole double-throw switch is available, it is advantageous to use two poles for reversing the battery connections and the other two for simultaneously reversing the galvanometer connections. This will preserve the sense of the galvanometer deflections in their relation to the unbalance of the bridge.

It is realized that rather elaborate precautions have been outlined in order to eliminate errors equivalent to a few thousandths, or at most hundredths, of a degree. Such precautions would hardly be justified in routine measurements, but since they will ordinarily be required only at the time of making a sulfur-point calibration, it is thought that some investigators may wish to make use of them.

The upper table of figure 2 indicates what values of effective seal resistance may be tolerated. A platinum resistance thermometer having an ice-point resistance of between 25 and $30 \mathrm{ohms}$ will have a sulfur-point resistance of the order of $75 \mathrm{ohms}$. If under the conditions of measurement the seal acts as a shunt having the resistance given in the first column of this table, the true resistance of the thermometer will be lowered by the amount given in the second column. Since for this type of thermometer $d t / d R$ is roughly 10 degrees per $\mathrm{ohm}$, the values in the last column are also approximately equal to the resulting error in thousandths of a degree.

The accuracy required in a sulfur-point calibration depends on the temperature interval in which the thermometer will subsequently be used. Suppose an error equivalent to 10 millidegrees is made in the sulfur-point calibration. Then the maximum resultant error [4] in the range $-182.97^{\circ}$ to $0^{\circ} \mathrm{C}$ is slightly less than 1 millidegree (near $-120^{\circ} \mathrm{C}$ ). In the range $0^{\circ}$ to $100^{\circ} \mathrm{C}$ the maximum resultant error (near $50^{\circ} \mathrm{C}$ ) is less than 0.2 millidegree. 


\section{SUPPLEMENTARY OBSERVATIONS}

The experimental results given in section II (and fig. 2) of this paper have been explained in terms of an increase of polarization of the glass caused by the passage of current. There is a great deal of experimental evidence in the literature showing that the electric current in glass is ordinarily carried by sodium ions, and that polarization results from a depletion of sodium ions in the layer of glass near the anode. Schtschukarew and Müller [5], among others, have shown that resistivity values reproducible both from sample to sample and from time to time may be obtained by using electrodes capable of supplying sodium ions to the glass. Robinson [6] has also demonstrated the necessity of avoiding polarization if true values of the resistivity of glass are to be obtained.

It was thought worth while to measure the resistivity of one of the types of glass used for glass seals under conditions where polarization would be largely avoided. The experimental arrangement used for this purpose is shown schematically in figure 3, together with the results obtained. The high temperatures involved made it impossible to use the sodium-amalgam electrodes which are ordinarily employed, so a sodium-tin alloy (roughly $3 \mathrm{Na}$ :97Sn by weight) was used. As this alloy must be protected from the air, it was used only for the anode, while pure tin served for the cathode. The inside tube of the apparatus was made of the glass to be tested, which was Corning normal bulb glass, the same as that used in seal 13 .

The curve at the left of the diagram was obtained by varying the applied electromotive force while the temperature was held constant at $445^{\circ} \mathrm{C}$. Because the two electrodes were of different composition, an electromotive force was produced, with pure tin positive relative to the alloy. As shown by the first point on the curve, it was necessary to oppose the electromotive force of this cell by one of approximately $1.4 \mathrm{v}$ to obtain zero current in an external circuit. As the opposing electromotive force was removed and replaced by one in the opposite direction, the current rapidly increased, while the effective electromotive force of the "cell" became much smaller. This is evident from the shape of the curve when it is recalled that if the cell developed no electromotive force, the curve would pass through the origin. The actual resistance of the specimen at $445^{\circ} \mathrm{C}$ was 432 ohms, which corresponds to a volume resistivity of the glass of approximately $21,000 \mathrm{ohm}-\mathrm{cm}$.

From the lower table of figure 2 we find that the effective resistance of a seal made from this type of glass ranged from 2.1 to 23 megohms, depending on the conditions of measurement. By employing these figures and estimates of the dimensions of the seal, calculations of the "effective resistivity" of the glass may be made. Such calculations yielded values from 8 to 90 times larger than the $21,000 \mathrm{ohm}-\mathrm{cm}$ found when polarization was avoided. The difference is to be attributed primarily to the effect of polarization. The term "effective resistance" has been applied to the quotient of the applied electromotive force by the observed current, to distinguish it from the true resistance in the absence of polarization.

A number of preliminary experiments performed on seals 1 to 12 also indicated that polarization is more important than resistivity of $446682-42-7$ 
the glass in determining the effective resistance. These seals consisted simply of two or more electrodes sealed into a small specimen of glass, and were measured under conditions less carefully controlled than those reported for seals 13, 14, and 15. Nevertheless it was plainly evident that the effective resistance was markedly affected by the material of the electrodes, and by the area in contact with the glass. Most of these seals had platinum electrodes, but one having constantan electrodes and another with silver electrodes had much lower effective resistances than the rest. In view of what is known about polarization, this is not surprising, for silver and copper will replace sodium in glass by electrolysis, while platinum will not [7]. Both the silver and the constantan discolored the glass somewhat when sealed in.

It is, of course, well known that a seal which has been polarized by the passage of current will serve as a source of electromotive force. In one of the preliminary experiments it was found that the initial charging by passage of current is not always necessary. In this experiment galvanometer deflections were produced by a freshly made seal to which no external electromotive force had ever been applied. The deflections corresponded to an electromotive force of the order of $0.1 \mathrm{v}$. Initial charges or polarization of this type could easily account for the directional dependence of the effective resistance found for seals 13,14, and 15 (difference between corresponding curves of dots and crosses in fig. 2). The seal whose initial charge was investigated showed a much larger electromotive force during its initial heating than on subsequent occasions. No appreciable electromotive force was detected below about $300^{\circ} \mathrm{C}$.

The apparatus shown in figure 3 was also used to obtain the temperature-dependence of the resistance of a specimen of glass. The dependence is shown by the semilog plot at the upper right in this figure. Both of the curves in figure 3 refer to the same specimen of Corning normal bulb glass. According to the theory of ionic conduction in solids [8, p. 235-241; and 9, p. 547-558], the passage of current is associated with the motion through the material of lattice imperfections, which may be either interstitial ions, vacancies at regular lattice points, or both. The current, which for ordinary field strengths should be proportional to the field, is also proportional to the number of lattice imperfections present and to the rate at which these imperfections wander through the material as a result of thermal agitation. Both of the two latter factors should vary exponentially with temperature. If $E$ is the nel energy required to form a lattice imperfection, and $U$ is the height of the potential barrier to be surmounted when an imperfection moves from one position of equilibrium to another, the conductance should be proportional to

$$
\exp -(1 / 2 E+U) / k T \text {. }
$$

Putting $\epsilon \equiv(1 / 2 E+U)$ the resistance of a specimen should be given by

$$
1 / r=A e-{ }^{\epsilon} / k T \text {. }
$$

The extent to which one is justified in applying a theory of this kind to glass is open to question. Since there is no long-range order in glass and only a limited amount of short-range order [10, 11], one might expect that the quantities $U$ and $E$ would not be sharply defined. However, it is of interest to see that the observed resistance of the glass does show a roughly exponential temperature-dependence. 
Taking logarithms of the equation above, we obtain

$$
\log r=-\log A+\frac{\epsilon}{2.303 k}\left(\frac{1}{T}\right)
$$

and by plotting $\log r$ against $1 / T$, we should be able to obtain $\epsilon$ from the slope. The experimental curve of figure 3 decreases in slope as the temperature increases. For comparison the dotted straight line, which has a slope corresponding to $\epsilon=0.8$ electron-volt, was drawn. This can be seen to be roughly equal to the mean slope of the experi-
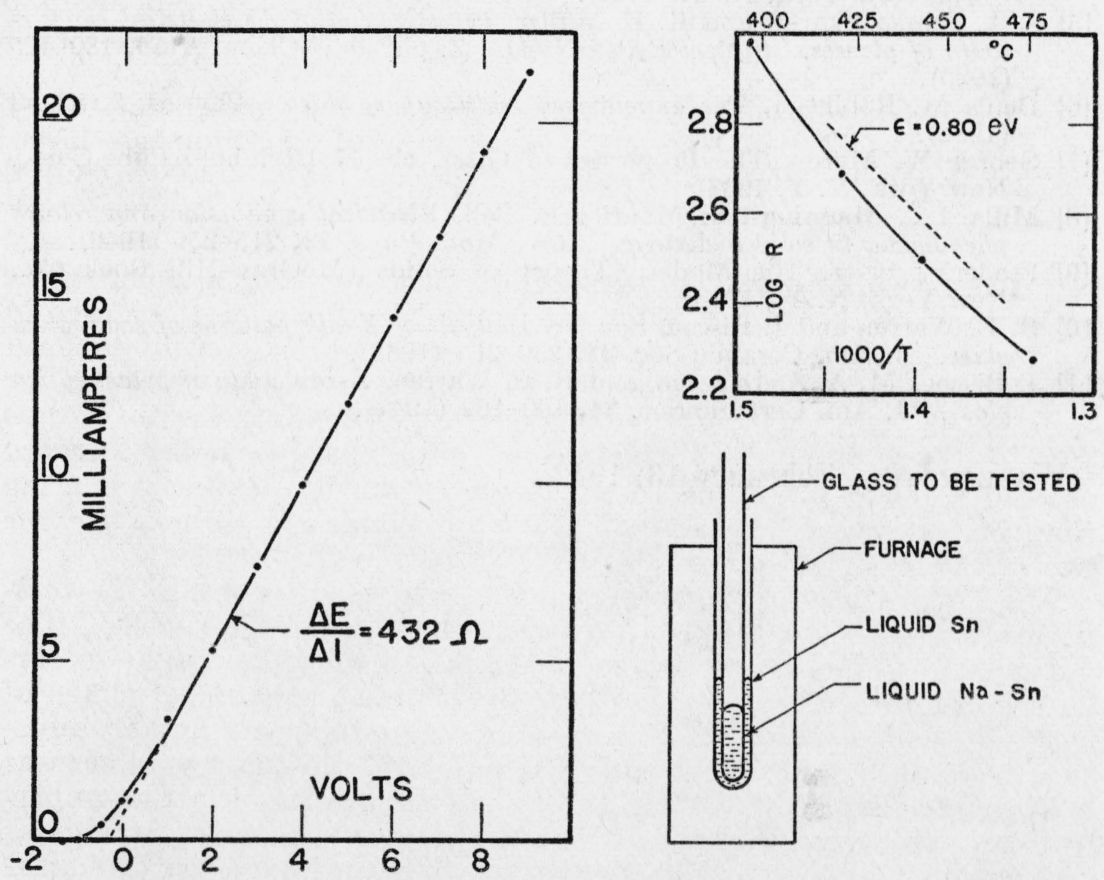

FiguRE 3.-Arrangement to eliminate polarization, and results obtained.

The curve at the left gives the variation of current with applied electromotive force, and shows that the "cell" develops an open-circuit electromotive force at $445^{\circ} \mathrm{C}$. of the order of $1.4 \mathrm{v}$. Pure tin is positive relative to the alloy, which had a composition of roughly $3 \mathrm{Na}: 97 \mathrm{Sn}$ by weight.

The curve at the upper right shows the temperature-dependence of the resistance of the glass when the electromotive force was held constant. The dotted line represents a slope corresponding to $\epsilon=0.8$ electron-volt in the equation $1 / r=A e^{-\triangleleft / k T}$.

mental curve. A suitable temperature-dependence of $U$ or $E$ could account for the observed curvature, or the curvature may be associated with the lack of sharply defined values of these quantities. According to Seitz [9, p. 549], the coefficient $A$ is proportional to $T^{2}$. The temperature variation in this factor is much less important than in the exponential factor. If $A$ is assumed to vary as $T^{2}$, the value obtained for $\epsilon$ in figure 3 is reduced by about 20 percent, without noticeable reduction in the curvature of the graph.

The author acknowledges valuable discussions with Frank Wenner, of the Resistance Measurements Section. 


\section{REFERENCES}

[1] J. C. Southard and R. T. Milner, Low temperature specific heats. II. The calibration of the thermometer and the resistance of $\mathrm{Pt}, \mathrm{Pt}-10 \% \mathrm{Rh}$, and constantan between -259 and $-190^{\circ}$. J. Am. Chem. Soc. 55, 4384-4391 (1933)

[2] George K. Burgess, The international temperature scale, BS J. Research 1, 635-640 (1928) RP22.

[3] Harold J. Hoge, Electrical conduction in the glass insulation of resistance thermometers, Phys. Rev. 59, 921 (1941) [abstract].

[4] E. F. Mueller, Precision Resistance Thermometry. Temperature, its Measurement and Control in Science and Industry, p. 162-179 (Reinhold Pub. Corp., New York, N. Y., 1941).

[5] S. A. Schtschukarew and R. L. Müller, Investigation of the electrical conductivity of glasses. System $\mathrm{B}_{2} \mathrm{O}_{3}+\mathrm{Na}_{2} \mathrm{O}$. Z . physik. Chem. A150, 439-475 (1930)

[6] Denis M. Robinson, The unpolarized resistivity of glass. Physics, 2, 52-59 (1932)

[7] George W. Morey, The Properties of Glass, ch. 17 (Reinhold Pub. Corp., New York, N. Y. 1938).

[8] Millard F. Manning and Maurice E. Bell, Electrical conduction and related phenomena in solid dielectrics. Rev. Mod. Phys. 12, 215-256 (1940).

[9] Frederick Seitz, The Modern Theory of Solids (McGraw-Hill Book Co., New York, N. Y., 1940).

10] B. E. Warren and J. Biscoe, Fourier Analysis of X-ray patterns of soda-silica glass. J. Am. Ceramic Soc. 21, 259-265 (1938).

[11] J. Biscoe, M. A. A. Druesne, and B. E. Warren, $X$-ray study of potash-silica glass. J. Am. Ceramic Soc. 24, 100-102 (1941).

Washington, February 13, 1942. 